\title{
Periodic solutions for a class of nonlinear difference equations
}

\author{
Haiping SHI, Xia LiU and Yuanbiao ZHANG
}

(Received December 2, 2013; Revised January 8, 2015)

\begin{abstract}
By using the critical point theory, some new criteria are obtained for the existence and multiplicity of periodic solutions to a class of nonlinear difference equations. The proof is based on the Linking Theorem in combination with variational technique. Our results successfully generalize and improve some existing results in the literature.

Key words: Periodic solutions, Nonlinear, Difference equations, Discrete variational theory.
\end{abstract}

\section{Introduction}

The problem of periodic solutions for differential equations has been the subject of many investigations [6], [14], [15], [28], [32], [33]. By using various methods and techniques, such as fixed point theory, the Kaplan-Yorke method, critical point theory, coincidence degree theory, bifurcation theory and dynamical system theory etc., a series of existence results for periodic solutions have been obtained in the literature. Difference equations, the discrete analogs of differential equations, occur widely in numerous settings and forms, both in mathematics itself and in its applications to statistics, computing, electrical circuit analysis, dynamical systems, economics, biology and other fields. For the general background of difference equations, one can refer to monographs [1], [3], [4], [25]. Since the past twenty years, there has been much progress on the qualitative properties of difference equations, which included results on stability and attractivity [16], [25], [27], [46] and results on oscillation and other topics [1]-[5], [9], [10], [21]-[23], [26], [31], [40], [41], [43]-[46]. Only a few papers discuss the periodic solutions of difference equations. Therefore, it is worthwhile to explore this topic.

2000 Mathematics Subject Classification : 39A23.

This project is supported by the Specialized Research Fund for Doctoral Program of Higher Eduction of China (No. 20114410110002), National Natural Science Foundation of China (No.11401121), Natural Science Foundation of Guangdong Province (No.S2013010014460) and Hunan Provincial Natural Science Foundation of China (No. 2015JJ2075). 
Below $\mathbf{N}, \mathbf{Z}$ and $\mathbf{R}$ denote the sets of all natural numbers, integers and real numbers respectively. For any $a, b \in \mathbf{Z}$, we denote $\mathbf{Z}(a)=\{a, a+$ $1, \ldots\}, \mathbf{Z}(a, b)=\{a, a+1, \ldots, b\}$ when $a \leq b$. Besides, * denotes the transpose of a vector.

The present paper considers the following forward and backward difference equation

$$
\Delta\left(p_{n}\left(\Delta u_{n-1}\right)^{\delta}\right)+f\left(n, u_{n+1}, u_{n}, u_{n-1}\right)=0, n \in \mathbf{Z}
$$

where $\Delta$ is the forward difference operator $\Delta u_{n}=u_{n+1}-u_{n}, \Delta^{2} u_{n}=$ $\Delta\left(\Delta u_{n}\right), \delta>0$ is the ratio of odd positive integers, $p_{n}$ is real valued for each $n \in \mathbf{Z}, f \in C\left(\mathbf{Z} \times \mathbf{R}^{3}, \mathbf{R}\right), p_{n}$ and $f\left(n, v_{1}, v_{2}, v_{3}\right)$ are $T$-periodic in $n$ for a given positive integer $T$.

Eq. (1.1) can be considered as a discrete analogue a special case of the following second-order nonlinear functional differential equation

$$
\left(p(t) \varphi\left(u^{\prime}\right)\right)^{\prime}+f(t, u(t+1), u(t), u(t-1))=0, t \in \mathbf{R} .
$$

Eq. (1.2) includes the following equation

$$
\left(p(t) \varphi\left(u^{\prime}\right)\right)^{\prime}+f(t, u(t))=0, t \in \mathbf{R},
$$

which has arose in the study of fluid dynamics, combustion theory, gas diffusion through porous media, thermal self-ignition of a chemically active mixture of gases in a vessel, catalysis theory, chemically reacting systems, and adiabatic reactor [8], [34]. Equations similar in structure to (1.2) arise in the study of the existence of solitary waves of lattice differential equations, see Smets and Willem [36].

When $\delta=1$, and $f\left(n, u_{n+1}, u_{n}, u_{n-1}\right)=q_{n} u_{n},(1.1)$ becomes

$$
\Delta\left(p_{n} \Delta u_{n-1}\right)+q_{n} u_{n}=0
$$

which has been extensively investigated by many authors [1], [4], [13], for results on oscillation, asymptotic behavior, boundary value problems, disconjugacy and disfocality.

When $f\left(n, u_{n+1}, u_{n}, u_{n-1}\right)=q_{n} u_{n}^{\delta}, n \in \mathbf{Z}(0)$, (1.1) reduces to the following equation 


$$
\Delta\left(p_{n}\left(\Delta u_{n-1}\right)^{\delta}\right)+q_{n} u_{n}^{\delta}=0
$$

which has been studied in [1], [11], [19], [37] for results on oscillation, asymptotic behavior and the existence of positive solutions.

Moreover, $f\left(n, u_{n+1}, u_{n}, u_{n-1}\right)=q_{n} g\left(u_{n}\right)+r_{n}$, (1.1) has been considered in [31], [37], [38] for oscillatory properties of its all solutions.

The widely used tools for the existence of periodic solutions of difference equations are the various fixed point theorems in cones [1], [3], [4], [25]. It is well known that critical point theory is a powerful tool that deals with the problems of differential equations [6], [8], [11], [18], [19], [28], [39]. Only since 2003, critical point theory has been employed to establish sufficient conditions on the existence of periodic solutions of difference equations. Recently, Guo and $\mathrm{Yu}$ [16]-[18] and Shi et al. [35] established sufficient conditions on the existence of periodic solutions of second-order nonlinear difference equations by using the critical point theory. In 2006, Cai, Yu and Guo [7] have obtained some sufficient conditions for the existence of periodic solutions of the following nonlinear difference equation

$$
\Delta\left(p_{n}\left(\Delta u_{n-1}\right)^{\delta}\right)+f\left(n, u_{n}\right)=0, n \in \mathbf{Z}
$$

However, to the best of our knowledge, when $\delta \neq 1$ the results on periodic solutions of nonlinear difference equation (1.1) are very scarce in the literature (see [7]), because there are few known methods for considering the existence of periodic solutions of discrete systems. Furthermore, since (1.1) contains both advance and retardation, there are very few manuscripts dealing with this subject. The main purpose of this paper is to give some sufficient conditions for the existence and multiplicity of periodic solutions to a class of nonlinear difference equations. The main approach used in our paper is a variational technique and the Linking Theorem. In particular, our results not only generalize the results in the literature [7], but also improve them. In fact, one can see the following Remarks 1.2 and 1.4 for details. The motivation for the present work stems from the recent papers in [12], [17].

Let

$$
\underline{p}=\min _{n \in \mathbf{Z}(1, T)}\left\{p_{n}\right\}, \bar{p}=\max _{n \in \mathbf{Z}(1, T)}\left\{p_{n}\right\}
$$

Our main results are as follows. 
Theorem 1.1 Assume that the following hypotheses are satisfied:

(p) $p_{n}>0, \forall n \in \mathbf{Z}$

$\left(F_{1}\right)$ there exists a functional $F\left(n, v_{1}, v_{2}\right) \in C^{1}\left(\mathbf{Z} \times \mathbf{R}^{2}, \mathbf{R}\right)$ with $F\left(n, v_{1}, v_{2}\right)$ $\geq 0$ and it satisfies

$$
\begin{aligned}
F\left(n+T, v_{1}, v_{2}\right) & =F\left(n, v_{1}, v_{2}\right), \\
\frac{\partial F\left(n-1, v_{2}, v_{3}\right)}{\partial v_{2}}+\frac{\partial F\left(n, v_{1}, v_{2}\right)}{\partial v_{2}} & =f\left(n, v_{1}, v_{2}, v_{3}\right) ;
\end{aligned}
$$

$\left(F_{2}\right)$ there exist constants $\eta_{1}>0, \alpha \in\left(0,\left(\underline{p} / 2^{(\delta+1) / 2}(\delta+1)\right)\left(c_{1} / c_{2}\right)^{\delta+1}\right.$ $\left.\cdot \lambda_{\min }^{(\delta+1) / 2}\right)$ such that

$$
F\left(n, v_{1}, v_{2}\right) \leq \alpha\left(\sqrt{v_{1}^{2}+v_{2}^{2}}\right)^{\delta+1}, \text { for } n \in \mathbf{Z} \text { and } v_{1}^{2}+v_{2}^{2} \leq \eta_{1}^{2} ;
$$

$\left(F_{3}\right)$ there exist constants $\rho_{1}>0, \zeta>0, \beta \in\left(\left(\bar{p} / 2^{(\delta+1) / 2}(\delta+1)\right)\left(c_{2} / c_{1}\right)^{\delta+1}\right.$ $\left.\cdot \lambda_{\max }^{(\delta+1) / 2},+\infty\right)$ such that

$$
F\left(n, v_{1}, v_{2}\right) \geq \beta\left(\sqrt{v_{1}^{2}+v_{2}^{2}}\right)^{\delta+1}-\zeta, \text { for } n \in \mathbf{Z} \text { and } v_{1}^{2}+v_{2}^{2} \geq \rho_{1}^{2},
$$

where $c_{1}, c_{2}$ are constants which can be referred to (2.4), and $\lambda_{\min }$, $\lambda_{\max }$ are constants which can be referred to (2.7).

Then (1.1) has at least three T-periodic solutions.

Remark 1.1 By $\left(F_{3}\right)$ it is easy to see that there exists a constant $\zeta^{\prime}>0$ such that $\left(F_{3}^{\prime}\right) F\left(n, v_{1}, v_{2}\right) \geq \beta\left(\sqrt{v_{1}^{2}+v_{2}^{2}}\right)^{\delta+1}-\zeta^{\prime}, \forall\left(n, v_{1}, v_{2}\right) \in \mathbf{Z} \times \mathbf{R}^{2}$.

As a matter of fact, let $\zeta_{1}=\max \left\{\left|F\left(n, v_{1}, v_{2}\right)-\beta\left(\sqrt{v_{1}^{2}+v_{2}^{2}}\right)^{\delta+1}+\zeta\right|\right.$ : $\left.n \in \mathbf{Z}, v_{1}^{2}+v_{2}^{2} \leq \rho_{1}^{2}\right\}, \zeta^{\prime}=\zeta+\zeta_{1}$, we can easily get the desired result.

Corollary 1.1 Assume that $(p)$ and $\left(F_{1}\right)-\left(F_{3}\right)$ are satisfied. Then (1.1) has at least two nontrivial T-periodic solutions.

Remark 1.2 Corollary 1.1 reduces to Theorem 3.1 in [7].

Theorem 1.2 Assume that $(p),\left(F_{1}\right)$ and the following conditions are 
satisfied:

$\left(F_{4}\right) \lim _{\rho \rightarrow 0} \frac{F\left(n, v_{1}, v_{2}\right)}{\rho^{\delta+1}}=0, \rho=\sqrt{v_{1}^{2}+v_{2}^{2}}, \forall n \in \mathbf{Z}$;

$\left(F_{5}\right)$ there exist constants $R_{1}>0$ and $\theta>\delta+1$ such that for $n \in \mathbf{Z}$ and $v_{1}^{2}+v_{2}^{2} \geq R_{1}^{2}$

$$
0<\theta F\left(n, v_{1}, v_{2}\right) \leq \frac{\partial F\left(n, v_{1}, v_{2}\right)}{\partial v_{1}} v_{1}+\frac{\partial F\left(n, v_{1}, v_{2}\right)}{\partial v_{2}} v_{2}
$$

Then (1.1) has at least three T-periodic solutions.

Remark 1.3 Assumption $\left(F_{5}\right)$ implies that there exist constants $a_{1}>0$ and $a_{2}>0$ such that

$\left(F_{5}^{\prime}\right) F\left(n, v_{1}, v_{2}\right) \geq a_{1}\left(\sqrt{v_{1}^{2}+v_{2}^{2}}\right)^{\theta}-a_{2}, \forall\left(n, v_{1}, v_{2}\right) \in \mathbf{Z} \times \mathbf{R}^{2}$.

Corollary 1.2 Assume that $(p)$ and $\left(F_{1}\right),\left(F_{4}\right),\left(F_{5}\right)$ are satisfied. Then (1.1) has at least two nontrivial T-periodic solutions.

If $f\left(n, u_{n+1}, u_{n}, u_{n-1}\right)=f\left(u_{n+1}, u_{n}, u_{n-1}\right),(1.1)$ reduces to the following nonlinear equation,

$$
\Delta\left(p_{n}\left(\Delta u_{n-1}\right)^{\delta}\right)+f\left(u_{n+1}, u_{n}, u_{n-1}\right)=0, n \in \mathbf{Z},
$$

where $f \in C\left(\mathbf{R}^{3}, \mathbf{R}\right)$. Then, we have the following results.

Theorem 1.3 Assume that $(p)$ and the following hypotheses are satisfied:

$\left(F_{6}\right)$ there exists a functional $F\left(v_{1}, v_{2}\right) \in C^{1}\left(\mathbf{R}^{2}, \mathbf{R}\right)$ with $F\left(v_{1}, v_{2}\right) \geq 0$ and it satisfies

$$
\frac{\partial F\left(v_{2}, v_{3}\right)}{\partial v_{2}}+\frac{\partial F\left(v_{1}, v_{2}\right)}{\partial v_{2}}=f\left(v_{1}, v_{2}, v_{3}\right)
$$

$\left(F_{7}\right)$ there exist constants $\eta_{2}>0, \alpha \in\left(0,\left(\underline{p} / 2^{(\delta+1) / 2}(\delta+1)\right)\left(c_{1} / c_{2}\right)^{\delta+1}\right.$ $\cdot \lambda_{\min }^{(\delta+1) / 2}$ ) such that

$$
F\left(v_{1}, v_{2}\right) \leq \alpha\left(\sqrt{v_{1}^{2}+v_{2}^{2}}\right)^{\delta+1}, \text { for } v_{1}^{2}+v_{2}^{2} \leq \eta_{2}^{2}
$$

$\left(F_{8}\right)$ there exist constants $\rho_{2}>0, \zeta>0, \beta \in\left(\left(\bar{p} / 2^{(\delta+1) / 2}(\delta+1)\right)\left(c_{2} / c_{1}\right)^{\delta+1}\right.$ 
$\left.\cdot \lambda_{\max }^{(\delta+1) / 2},+\infty\right)$ such that

$$
F\left(v_{1}, v_{2}\right) \geq \beta\left(\sqrt{v_{1}^{2}+v_{2}^{2}}\right)^{\delta+1}-\zeta, \text { for } v_{1}^{2}+v_{2}^{2} \geq \rho_{2}^{2}
$$

where $c_{1}, c_{2}$ are constants which can be referred to (2.4), and $\lambda_{\min }$, $\lambda_{\max }$ are constants which can be referred to (2.7).

Then (1.6) has at least three T-periodic solutions.

Corollary 1.3 Assume that $(p)$ and $\left(F_{6}\right)-\left(F_{8}\right)$ are satisfied. Then (1.6) has at least two nontrivial T-periodic solutions.

Remark 1.4 Corollary 1.3 reduces to Theorem 3.2 in [7].

The rest of the paper is organized as follows. In Section 2, we shall establish the variational framework associated with (1.1) and transfer the problem of the existence of periodic solutions of (1.1) into that of the existence of critical points of the corresponding functional. Some related fundamental results will also be recalled. In Section 3, we shall complete the proof of the results by using the critical point method.

For the basic knowledge of variational methods, the reader is referred to $[15],[23],[24],[29]$.

\section{Variational structure and some lemmas}

Let $S$ be the set of sequences $u=\left(\ldots, u_{-n}, \ldots, u_{-1}, u_{0}, u_{1}, \ldots, u_{n}, \ldots\right)$ $=\left\{u_{n}\right\}_{n=-\infty}^{+\infty}$, that is

$$
S=\left\{\left\{u_{n}\right\} \mid u_{n} \in \mathbf{R}, n \in \mathbf{Z}\right\} .
$$

For any given positive integer $T, E_{T}$ is defined as a subspace of $S$ by

$$
E_{T}=\left\{u \in S \mid u_{n+T}=u_{n}, \forall n \in \mathbf{Z}\right\} .
$$

Clearly, $E_{T}$ is isomorphic to $\mathbf{R}^{T} . E_{T}$ can be equipped with the inner product

$$
\langle u, v\rangle=\sum_{j=1}^{T} u_{j} v_{j}, \forall u, v \in E_{T},
$$

by which the norm $\|\cdot\|$ can be induced by 


$$
\|u\|=\left(\sum_{j=1}^{T} u_{j}^{2}\right)^{1 / 2}, \forall u \in E_{T} .
$$

It is obvious that $E_{T}$ with the inner product (2.1) is a finite dimensional Hilbert space and linearly homeomorphic to $\mathbf{R}^{T}$.

On the other hand, we define the norm $\|\cdot\|_{s}$ on $E_{T}$ as follows:

$$
\|u\|_{s}=\left(\sum_{j=1}^{T}\left|u_{j}\right|^{s}\right)^{1 / s}
$$

for all $u \in E_{T}$ and $s>1$.

Since $\|u\|_{s}$ and $\|u\|_{2}$ are equivalent, there exist constants $c_{1}, c_{2}$ such that $c_{2} \geq c_{1}>0$, and

$$
c_{1}\|u\|_{2} \leq\|u\|_{s} \leq c_{2}\|u\|_{2}, \forall u \in E_{T}
$$

Clearly, $\|u\|=\|u\|_{2}$. For all $u \in E_{T}$, define the functional $J$ on $E_{T}$ as follows:

$$
J(u)=\frac{1}{\delta+1} \sum_{n=1}^{T} p_{n}\left(\Delta u_{n-1}\right)^{\delta+1}-\sum_{n=1}^{T} F\left(n, u_{n+1}, u_{n}\right)
$$

where

$$
\frac{\partial F\left(n-1, v_{2}, v_{3}\right)}{\partial v_{2}}+\frac{\partial F\left(n, v_{1}, v_{2}\right)}{\partial v_{2}}=f\left(n, v_{1}, v_{2}, v_{3}\right)
$$

Clearly, $J \in C^{1}\left(E_{T}, \mathbf{R}\right)$. Due to the periodicity of $u=\left\{u_{n}\right\}_{n \in \mathbf{Z}} \in E_{T}$ and $f\left(n, v_{1}, v_{2}, v_{3}\right)$ in the first variable $n$, we reduce the existence of periodic solutions of (1.1) to the existence of critical points of $J$ on $E_{T}$. That is, the functional $J$ is just the variational framework of (1.1).

Let $P$ be the $T \times T$ matrix defined by

$$
P=\left(\begin{array}{cccccc}
2 & -1 & 0 & \ldots & 0 & -1 \\
-1 & 2 & -1 & \ldots & 0 & 0 \\
0 & -1 & 2 & \ldots & 0 & 0 \\
\ldots & \ldots & \ldots & \ldots & \ldots & \ldots \\
0 & 0 & 0 & \ldots & 2 & -1 \\
-1 & 0 & 0 & \ldots & -1 & 2
\end{array}\right)
$$


By matrix theory [42], we see that the eigenvalues of $P$ are

$$
\lambda_{j}=2\left(1-\cos \frac{2 j}{T} \pi\right), j=0,1,2, \ldots, T-1 .
$$

Thus, $\lambda_{0}=0, \lambda_{1}>0, \lambda_{2}>0, \ldots, \lambda_{T-1}>0$. Therefore,

$$
\begin{aligned}
& \lambda_{\min }=\min \left\{\lambda_{1}, \lambda_{2}, \ldots, \lambda_{T-1}\right\}=2\left(1-\cos \frac{2}{T} \pi\right), \\
& \lambda_{\max }=\max \left\{\lambda_{1}, \lambda_{2}, \ldots, \lambda_{T-1}\right\}=\left\{\begin{array}{ll}
4, & \text { when } T \text { is even, } \\
2\left(1+\cos \frac{1}{T} \pi\right), & \text { when } T \text { is odd. }
\end{array}\right\}
\end{aligned}
$$

Let

$$
W=\operatorname{ker} P=\left\{u \in E_{T} \mid P u=0 \in \mathbf{R}^{T}\right\} .
$$

Then

$$
W=\left\{u \in E_{T} \mid u=\{c\}, c \in \mathbf{R}\right\}
$$

Let $V$ be the direct orthogonal complement of $E_{T}$ to $W$, i.e., $E_{T}=$ $V \oplus W$. For convenience, we identify $u \in E_{T}$ with $u=\left(u_{1}, u_{2}, \ldots, u_{T}\right)^{*}$.

Let $E$ be a real Banach space, $J \in C^{1}(E, \mathbf{R})$, i.e., $J$ is a continuously Fréchet-differentiable functional defined on $E$. $J$ is said to satisfy the PalaisSmale condition (P.S. condition for short) if any sequence $\left\{u^{(i)}\right\} \subset E$ for which $\left\{J\left(u^{(i)}\right)\right\}$ is bounded and $J^{\prime}\left(u^{(i)}\right) \rightarrow 0(i \rightarrow \infty)$ possesses a convergent subsequence in $E$.

Let $B_{\rho}$ denote the open ball in $E$ about 0 of radius $\rho$ and let $\partial B_{\rho}$ denote its boundary.

Lemma 2.1 (Linking Theorem [34]) Let $E$ be a real Banach space, $E=$ $E_{1} \oplus E_{2}$, where $E_{1}$ is finite dimensional. Suppose that $J \in C^{1}(E, \mathbf{R})$ satisfies the P.S. condition and

$\left(J_{1}\right)$ there exist constants $a>0$ and $\rho>0$ such that $\left.J\right|_{\partial B_{\rho} \cap E_{2}} \geq a$;

$\left(J_{2}\right)$ there exists an $e \in \partial B_{1} \cap E_{2}$ and a constant $R_{0} \geq \rho$ such that $\left.J\right|_{\partial Q} \leq 0$, where $Q=\left(\bar{B}_{R_{0}} \cap E_{1}\right) \oplus\left\{s e \mid 0<s<R_{0}\right\}$. 
Then $J$ possesses a critical value $c \geq a$, where

$$
c=\inf _{h \in \Gamma} \sup _{u \in Q} J(h(u)),
$$

and $\Gamma=\left\{h \in C(\bar{Q}, E)|h|_{\partial Q}=i d\right\}$, where $i d$ denotes the identity operator.

Lemma 2.2 Assume that $(p),\left(F_{1}\right)$ and $\left(F_{3}\right)$ are satisfied. Then the functional $J$ is bounded from above in $E_{T}$.

Proof. By $\left(F_{3}^{\prime}\right)$ and $(2.4)$, for any $u \in E_{T}$,

$$
\begin{aligned}
J(u)= & \frac{1}{\delta+1} \sum_{n=1}^{T} p_{n}\left(\Delta u_{n-1}\right)^{\delta+1}-\sum_{n=1}^{T} F\left(n, u_{n+1}, u_{n}\right) \\
\leq & \frac{\bar{p}}{\delta+1} \sum_{n=1}^{T}\left|\Delta u_{n}\right|^{\delta+1}-\sum_{n=1}^{T} F\left(n, u_{n+1}, u_{n}\right) \\
= & \frac{\bar{p}}{\delta+1}\left[\left(\sum_{n=1}^{T}\left|\Delta u_{n}\right|^{\delta+1}\right)^{1 /(\delta+1)}\right]^{\delta+1}-\sum_{n=1}^{T} F\left(n, u_{n+1}, u_{n}\right) \\
\leq & \frac{\bar{p}}{\delta+1}\left[c_{2}\left(\sum_{n=1}^{T}\left|\Delta u_{n}\right|^{2}\right)^{1 / 2}\right]^{\delta+1}-\sum_{n=1}^{T}\left[\beta\left(\sqrt{u_{n+1}^{2}+u_{n}^{2}}\right)^{\delta+1}-\zeta^{\prime}\right] \\
= & \frac{\bar{p}}{\delta+1} c_{2}^{\delta+1}\left[\sum_{n=1}^{T} 2\left(u_{n}^{2}-u_{n} u_{n+1}\right)\right]^{(\delta+1) / 2} \\
& -\beta\left[\left(\sum_{n=1}^{T}\left(\sqrt{\left.u_{n+1}^{2}+u_{n}^{2}\right)}\right)^{\delta+1}\right)^{1 /(\delta+1)}\right]^{\delta+1}+T \zeta^{\prime} \\
\leq & \frac{\bar{p}}{\delta+1} c_{2}^{\delta+1}\left[\sum_{n=1}^{T+1} 2\left(u_{n}^{2}-u_{n}^{*} u_{n+1}\right)\right]^{(\delta+1) / 2} \\
& -\beta c_{1}^{\delta+1}\left[\sum_{n=1}^{T}\left(u_{n+1}^{2}+u_{n}^{2}\right)\right]^{(\delta+1) / 2}+T \zeta^{\prime} \\
&
\end{aligned}
$$




$$
\begin{aligned}
& \leq \frac{\bar{p}}{\delta+1} c_{2}^{\delta+1} \lambda_{\max }^{(\delta+1) / 2}\|u\|_{2}^{\delta+1}-2^{(\delta+1) / 2} \beta c_{1}^{\delta+1}\|u\|_{2}^{\delta+1}+T \zeta^{\prime} \\
& =\left(\frac{\bar{p}}{\delta+1} c_{2}^{\delta+1} \lambda_{\max }^{(\delta+1) / 2}-2^{(\delta+1) / 2} \beta c_{1}^{\delta+1}\right)\|u\|_{2}^{\delta+1}+T \zeta^{\prime} \\
& \leq T \zeta^{\prime}
\end{aligned}
$$

The proof of Lemma 2.2 is complete.

Remark 2.1 The case $T=1$ is trivial. For the case $T=2, P$ has a different form, namely,

$$
P=\left(\begin{array}{cc}
2 & -2 \\
-2 & 2
\end{array}\right)
$$

However, in this special case, the argument need not to be changed and we omit it.

Lemma 2.3 Assume that $(p),\left(F_{1}\right)$ and $\left(F_{3}\right)$ are satisfied. Then the functional $J$ satisfies the P.S. condition.

Proof. Let $\left\{J\left(u^{(i)}\right)\right\}$ be a bounded sequence from the lower bound, i.e., there exists a positive constant $M_{1}$ such that

$$
-M_{1} \leq J\left(u^{(i)}\right), \forall i \in \mathbf{N}
$$

By the proof of Lemma 2.2, it is easy to see that

$$
\begin{array}{r}
-M_{1} \leq J\left(u^{(i)}\right) \leq\left(\frac{\bar{p}}{\delta+1} c_{2}^{\delta+1} \lambda_{\max }^{(\delta+1) / 2}-2^{(\delta+1) / 2} \beta c_{1}^{\delta+1}\right)\left\|u^{(i)}\right\|_{2}^{\delta+1}+T \zeta^{\prime} \\
\forall i \in \mathbf{N} .
\end{array}
$$

Therefore,

$$
\left(2^{(\delta+1) / 2} \beta c_{1}^{\delta+1}-\frac{\bar{p}}{\delta+1} c_{2}^{\delta+1} \lambda_{\max }^{(\delta+1) / 2}\right)\left\|u^{(i)}\right\|_{2}^{\delta+1} \leq M_{1}+T \zeta^{\prime} .
$$

Since $\beta>\left(\bar{p} / 2^{(\delta+1) / 2}(\delta+1)\right)\left(c_{2} / c_{1}\right)^{\delta+1} \lambda_{\max }^{(\delta+1) / 2}$, it is not difficult to know that $\left\{u^{(i)}\right\}$ is a bounded sequence in $E_{T}$. As a consequence, $\left\{u^{(i)}\right\}$ possesses a convergence subsequence in $E_{T}$. Thus the P.S. condition is verified. 


\section{Proof of the main results}

In this Section, we shall prove our main results by using the critical point method.

\subsection{Proof of Theorem 1.1}

Assumptions $\left(F_{1}\right)$ and $\left(F_{2}\right)$ imply that $F(n, 0)=0$ and $f(n, 0)=0$ for $n \in \mathbf{Z}$. Then $u=0$ is a trivial $T$-periodic solution of (1.1).

By Lemma 2.2, $J$ is bounded from the upper on $E_{T}$. We define $c_{0}=$ $\sup _{u \in E_{T}} J(u)$. The proof of Lemma 2.2 implies $\lim _{\|u\|_{2} \rightarrow+\infty} J(u)=-\infty$. This means that $-J(u)$ is coercive. By the continuity of $J(u)$, there exists $\bar{u} \in E_{T}$ such that $J(\bar{u})=c_{0}$. Clearly, $\bar{u}$ is a critical point of $J$.

We claim that $c_{0}>0$. Indeed, by $\left(F_{2}\right)$, for any $u \in V,\|u\|_{2} \leq \eta_{1}$, we have

$$
\begin{aligned}
J(u)= & \frac{1}{\delta+1} \sum_{n=1}^{T} p_{n}\left(\Delta u_{n-1}\right)^{\delta+1}-\sum_{n=1}^{T} F\left(n, u_{n+1}, u_{n}\right) \\
\geq & \frac{\underline{p}}{\delta+1} \sum_{n=1}^{T}\left|\Delta u_{n}\right|^{\delta+1}-\sum_{n=1}^{T} F\left(n, u_{n+1}, u_{n}\right) \\
= & \frac{\underline{p}}{\delta+1}\left[\left(\sum_{n=1}^{T}\left|\Delta u_{n}\right|^{\delta+1}\right)^{1 /(\delta+1)}\right]^{\delta+1}-\sum_{n=1}^{T} F\left(n, u_{n+1}, u_{n}\right) \\
\geq & \frac{\underline{p}}{\delta+1}\left[c_{1}\left(\sum_{n=1}^{T}\left|\Delta u_{n}\right|^{2}\right)^{1 / 2}\right]^{\delta+1}-\alpha \sum_{n=1}^{T}\left(\sqrt{u_{n+1}^{2}+u_{n}^{2}}\right) \\
= & \frac{\underline{p}}{\delta+1} c_{1}^{\delta+1}\left[\sum_{n=1}^{T} 2\left(u_{n}^{2}-u_{n} u_{n+1}\right)\right]^{(\delta+1) / 2} \\
& -\alpha\left[\left(\sum _ { n = 1 } ^ { T } \left(\sqrt{\left.\left.\left.u_{n+1}^{2}+u_{n}^{2}\right)^{\delta+1}\right)^{1 /(\delta+1)}\right]^{\delta+1}}\right.\right.\right. \\
\geq & \frac{-\alpha}{\delta+1} c_{1}^{\delta+1}\left[\sum_{n=1}^{T+1} 2\left(\sum_{n=1}^{2}-u_{n} u_{n+1}\left(u_{n+1}^{2}+u_{n}^{2}\right)\right]^{(\delta+1) / 2}\right.
\end{aligned}
$$




$$
\begin{aligned}
& =\frac{\underline{p}}{\delta+1} c_{1}^{\delta+1}\left(u^{*} P u\right)^{(\delta+1) / 2}-\alpha c_{2}^{\delta+1}\left(2\|u\|_{2}^{2}\right)^{(\delta+1) / 2} \\
& \geq \frac{\underline{p}}{\delta+1} c_{1}^{\delta+1} \lambda_{\min }^{(\delta+1) / 2}\|u\|_{2}^{\delta+1}-2^{(\delta+1) / 2} \alpha c_{2}^{\delta+1}\|u\|_{2}^{\delta+1} \\
& =\left(\frac{\underline{p}}{\delta+1} c_{1}^{\delta+1} \lambda_{\min }^{(\delta+1) / 2}-2^{(\delta+1) / 2} \alpha c_{2}^{\delta+1}\right)\|u\|_{2}^{\delta+1}
\end{aligned}
$$

Take $\sigma=\left((\underline{p} /(\delta+1)) c_{1}^{\delta+1} \lambda_{\min }^{(\delta+1) / 2}-2^{(\delta+1) / 2} \alpha c_{2}^{\delta+1}\right) \eta_{1}^{\delta+1}$. Then

$$
J(u) \geq \sigma, \forall u \in V \cap \partial B_{\eta_{1}} .
$$

Therefore, $c_{0}=\sup _{u \in E_{T}} J(u) \geq \sigma>0$. At the same time, we have also proved that there exist constants $\sigma>0$ and $\eta_{1}>0$ such that $\left.J\right|_{\partial B_{\eta_{1}} \cap V} \geq \sigma$. That is to say, $J$ satisfies the condition $\left(J_{1}\right)$ of the Linking Theorem.

Noting that $\sum_{n=1}^{T} p_{n}\left(\Delta u_{n-1}\right)^{\delta+1}=0$, for all $u \in W$, we have

$$
\begin{aligned}
J(u) & =\frac{1}{\delta+1} \sum_{n=1}^{T} p_{n}\left(\Delta u_{n-1}\right)^{\delta+1}-\sum_{n=1}^{T} F\left(n, u_{n+1}, u_{n}\right) \\
& =-\sum_{n=1}^{T} F\left(n, u_{n+1}, u_{n}\right) \leq 0 .
\end{aligned}
$$

Thus, the critical point $\bar{u}$ of $J$ corresponding to the critical value $c_{0}$ is a nontrivial $T$-periodic solution of (1.1).

In order to obtain another nontrivial $T$-periodic solution of (1.1) different from $\bar{u}$, we need to use the conclusion of Lemma 2.1. We have known that $J$ satisfies the P.S. condition on $E_{T}$. In the following, we shall verify the condition $\left(J_{2}\right)$.

Take $e \in \partial B_{1} \cap V$, for any $z \in W$ and $r \in \mathbf{R}$, let $u=r e+z$. Then

$$
\begin{aligned}
J(u) & =\frac{1}{\delta+1} \sum_{n=1}^{T} p_{n+1}\left(\Delta u_{n}\right)^{\delta+1}-\sum_{n=1}^{T} F\left(n, u_{n+1}, u_{n}\right) \\
& \leq \frac{\bar{p}}{\delta+1} \sum_{n=1}^{T}\left[\left|r \Delta e_{n}\right|^{\delta+1}-F\left(n, r e_{n+1}+z_{n+1}, r e_{n}+z_{n}\right)\right]
\end{aligned}
$$




$$
\begin{aligned}
& \leq \frac{\bar{p}}{\delta+1} r^{\delta+1}\left[\left(\sum_{n=1}^{T}\left|\Delta e_{n}\right|^{\delta+1}\right)^{1 /(\delta+1)}\right]^{\delta+1} \\
& -\sum_{n=1}^{T}\left\{\beta\left(\sqrt{\left(r e_{n+1}+z_{n+1}\right)^{2}+\left(r e_{n}+z_{n}\right)^{2}}\right)^{\delta+1}-\zeta^{\prime}\right\} \\
& \leq \frac{\bar{p}}{\delta+1} r^{\delta+1} c_{2}^{\delta+1}\left(\sum_{n=1}^{T}\left|\Delta e_{n}\right|^{2}\right)^{(\delta+1) / 2} \\
& -\beta c_{1}^{\delta+1}\left\{\sum_{n=1}^{T}\left[\left(r e_{n+1}+z_{n+1}\right)^{2}+\left(r e_{n}+z_{n}\right)^{2}\right]\right\}^{(\delta+1) / 2}+T \zeta^{\prime} \\
& =\frac{\bar{p}}{\delta+1} r^{\delta+1} c_{2}^{\delta+1}\left[\sum_{n=1}^{T} 2\left(e_{n}^{2}-e_{n+1} e_{n}\right)\right]^{(\delta+1) / 2} \\
& -\beta c_{1}^{\delta+1}\left\{\sum_{n=1}^{T}\left[\left(r e_{n+1}+z_{n+1}\right)^{2}+\left(r e_{n}+z_{n}\right)^{2}\right]\right\}^{(\delta+1) / 2}+T \zeta^{\prime} \\
& \leq \frac{\bar{p}}{\delta+1} r^{\delta+1} c_{2}^{\delta+1} \lambda_{\max }^{(\delta+1) / 2}-\beta c_{1}^{\delta+1}\left[2 \sum_{n=1}^{T}\left(r e_{n}+z_{n}\right)^{2}\right]^{(\delta+1) / 2}+T \zeta^{\prime} \\
& =\frac{\bar{p}}{\delta+1} r^{\delta+1} c_{2}^{\delta+1} \lambda_{\max }^{(\delta+1) / 2}-\beta c_{1}^{\delta+1} r^{\delta+1} 2^{(\delta+1) / 2} \\
& -\beta c_{1}^{\delta+1} 2^{(\delta+1) / 2}\|z\|_{2}^{\delta+1}+T \zeta^{\prime} \\
& =\left(\frac{\bar{p}}{\delta+1} c_{2}^{\delta+1} \lambda_{\max }^{(\delta+1) / 2}-\beta c_{1}^{\delta+1} 2^{(\delta+1) / 2}\right) r^{\delta+1} \\
& -\beta c_{1}^{\delta+1} 2^{(\delta+1) / 2}\|z\|_{2}^{\delta+1}+T \zeta^{\prime} \\
& \leq-\beta c_{1}^{\delta+1} 2^{(\delta+1) / 2}\|z\|_{2}^{\delta+1}+T \zeta^{\prime} \text {. }
\end{aligned}
$$

Thus, there exists a positive constant $R_{2}>\eta_{1}$ such that for any $u \in \partial Q$, $J(u) \leq 0$, where $Q=\left(\bar{B}_{R_{2}} \cap W\right) \oplus\left\{r e \mid 0<r<R_{2}\right\}$. By the Linking Theorem, $J$ possesses a critical value $c \geq \sigma>0$, where

$$
c=\inf _{h \in \Gamma} \sup _{u \in Q} J(h(u))
$$


and $\Gamma=\left\{h \in C\left(\bar{Q}, E_{T}\right)|h|_{\partial Q}=i d\right\}$.

Let $\tilde{u} \in E_{T}$ be a critical point associated to the critical value $c$ of $J$, i.e., $J(\tilde{u})=c$. If $\tilde{u} \neq \bar{u}$, then the conclusion of Theorem 1.1 holds. Otherwise, $\tilde{u}=\bar{u}$. Then $c_{0}=J(\bar{u})=J(\tilde{u})=c$, that is $\sup _{u \in E_{T}} J(u)=$ $\inf _{h \in \Gamma} \sup _{u \in Q} J(h(u))$. Choosing $h=i d$, we have $\sup _{u \in Q} J(u)=c_{0}$. Since the choice of $e \in \partial B_{1} \cap V$ is arbitrary, we can take $-e \in \partial B_{1} \cap V$. Similarly, there exists a positive number $R_{3}>\eta_{1}$, for any $u \in \partial Q_{1}, J(u) \leq 0$, where $Q_{1}=\left(\bar{B}_{R_{3}} \cap W\right) \oplus\left\{-r e \mid 0<r<R_{3}\right\}$.

Again, by the Linking Theorem, $J$ possesses a critical value $c^{\prime} \geq \sigma>0$, where

$$
c^{\prime}=\inf _{h \in \Gamma_{1}} \sup _{u \in Q_{1}} J(h(u)),
$$

and $\Gamma_{1}=\left\{h \in C\left(\bar{Q}_{1}, E_{T}\right)|h|_{\partial Q_{1}}=i d\right\}$.

If $c^{\prime} \neq c_{0}$, then the proof is finished. If $c^{\prime}=c_{0}$, then $\sup _{u \in Q_{1}} J(u)=c_{0}$. Due to the fact $\left.J\right|_{\partial Q} \leq 0$ and $\left.J\right|_{\partial Q_{1}} \leq 0, J$ attains its maximum at some points in the interior of sets $Q$ and $Q_{1}$. However, $Q \cap Q_{1} \subset W$ and $J(u) \leq 0$ for any $u \in W$. Therefore, there must be a point $u^{\prime} \in E_{T}, u^{\prime} \neq \tilde{u}$ and $J\left(u^{\prime}\right)=c^{\prime} \neq c_{0}$. The proof of Theorem 1.1 is complete.

Remark 3.1 Similarly to above argument, we can also prove Theorems 1.2 and 1.3. For simplicity, we omit their proofs.

Remark 3.2 Due to Theorems 1.1, 1.2 and 1.3, the conclusion of Corollaries 1.1, 1.2 and 1.3 is obviously true.

\section{References}

[ 1 ] Agarwal R. P., Difference Equations and Inequalities: Theory, Methods and Applications. Marcel Dekker, New York (1992).

[ 2 ] Agarwal R. P., Perera K. and O'regan D., Multiple positive solutions of singular and nonsingular discrete problems via variational methods. Nonlinear Anal. 58 (2004), 69-73.

[ 3 ] Agarwal R. P. and Wong P. J. Y., Advanced Topics in Difference Equations. Kluwer Academic Publishers, Dordrecht (1997).

[ 4 ] Ahlbrandt C. D. and Peterson A. C., Discrete Hamiltonian Systems: Difference Equations, Continued Fraction and Riccati Equations. Kluwer Academic Publishers, Dordrecht (1996).

[5] Avery R. I. and Pererson A. C., Three positive fixed points of nonlinear 
operators on ordered Banach space. Comput. Math. Appl. 42 (2001), 313322.

[ 6 ] Benci V. and Rabinowitz P. H., Critical point theorems for indefinite functionals. Invent Math. 52 (1979), 241-273.

[ 7 ] Cai X. C., Yu J. S. and Guo Z. M., Periodic solutions of a class of nonlinear difference equations via critical point method. Comput. Math. Appl. 52 (2006), 1639-1647.

[ 8 ] Chang K. C., Infinite Dimensional Morse Theory and Multiple Solution Problems. Birkhäuser, Boston (1993).

[ 9 ] Chen P. and Fang H., Existence of periodic and subharmonic solutions for second-order p-Laplacian difference equations. Adv. Difference Equ. 2007 (2007), 1-9.

[10] Chen P. and Tang X. H., Existence of infinitely many homoclinic orbits for fourth-order difference systems containing both advance and retardation. Appl. Math. Comput. 217 (2011), 4408-4415.

[11] Chen P. and Tang X. H., New existence and multiplicity of solutions for some Dirichlet problems with impulsive effects. Math. Comput. Modelling 55 (2012), 723-739.

[12] Chen P. and Tang X. H., Existence of homoclinic solutions for some secondorder discrete Hamiltonian systems. J. Difference Equ. Appl. 19 (2013), 633-648.

[13] Chen S. Z., Disconjugacy, disfocality, and oscillation of second order difference equation. J. Differential Equations 107 (1994), 383-394.

[14] Clarke F. H., Periodic solutions to Hamiltonian inclusions. J. Differential Equations, 40 (1981), 1-6.

[15] Cordaro G., Existence and location of periodic solution to convex and non coercive Hamiltonian systems. Discrete Contin. Dyn. Syst. 12 (2005), 983996.

[16] Erbe L.H., Xia H. and Yu J. S., Global stability of a linear nonautonomous delay difference equations. J. Difference Equ. Appl. 1 (1995), 151-161.

[17] Guo C. J., O'Regan D. and Agarwal R. P., Existence of multiple periodic solutions for a class of first-order neutral differential equations. Appl. Anal. Discrete Math. 5 (2011), 147-158.

[18] Guo C. J., O'Regan D., Xu Y. T. and Agarwal R. P., Existence and multiplicity of homoclinic orbits of a second-order differential difference equation via variational methods. Appl. Math. Inform. Mech. 4 (2012), 1-15.

[19] Guo C. J. and Xu Y. T., Existence of periodic solutions for a class of second order differential equation with deviating argument. J. Appl. Math. Comput. 28 (2008), 425-433. 
[20] Guo D. J., Nonlinear Functional Analysis. Shandong Scientific Press, Jinan (1985).

[21] Guo Z. M. and Yu J. S., Applications of critical point theory to difference equations. Fields Inst. Commun. 42 (2004), 187-200.

[22] Guo Z. M. and Yu J. S., Existence of periodic and subharmonic solutions for second-order superlinear difference equations. Sci. China Math. 46 (2003), 506-515.

[23] Guo Z. M. and Yu J. S., The existence of periodic and subharmonic solutions of subquadratic second order difference equations. J. London Math. Soc. 68 (2003), 419-430.

[24] He X. Z., Oscillatory and asymptotic behavior of second order nonlinear difference equations. J. Math. Anal. Appl. 175 (1993), 482-498.

[25] Kocic V. L. and Ladas G., Global Behavior of Nonlinear Difference Equations of Higher Order with Applications. Kluwer Academic Publishers, Dordrecht (1993).

[26] Liu Y. J. and Ge W. G., Twin positive solutions of boundary value problems for finite difference equations with p-Laplacian operator. J. Math. Appl. 278 (2003), 551-561.

[27] Matsunaga H., Hara T. and Sakata S., Global attractivity for a nonlinear difference equation with variable delay. Computers Math. Appl. 41 (2001), 543-551.

[28] Mawhin J. and Willem M., Critical Point Theory and Hamiltonian Systems. Springer, New York (1989).

[29] Pankov A. and Zakhrchenko N., On some discrete variational problems. Acta Appl. Math. 65 (2001), 295-303.

[30] Peil T. and Peterson A., Asymptotic behavior of solutions of a two-term difference equation. Rocky Mountain J. Math. 24 (1994), 233-251.

[31] Peng M. S., Xu Q. L., Huang L. H., et al., Asymptotic and oscillatory behavior of solutions of certain second order nonlinear difference equations. Comput. Math. Appl. 37 (1999), 9-18.

[32] Rabinowitz P. H., Periodic solutions of Hamiltonian systems. Comm. Pure Appl. Math. 31 (1978), 157-184.

[33] Rabinowitz P. H., On subharmonic solutions of Hamiltonian systems. Comm. Pure Appl. Math. 33 (1980), 609-633.

[34] Rabinowitz P.H., Minimax Methods in Critical Point Theory with Applications to Differential Equations. Amer. Math. Soc., Providence, RI, New York (1986).

[35] Shi H. P., Ling W. P., Long Y. H. and Zhang H. Q., Periodic and subharmonic solutions for second order nonlinear functional difference equations. 
Commun. Math. Anal. 5 (2008), 50-59.

[36] Smets D. and Willem M., Solitary waves with prescribed speed on infinite lattices. J. Funct. Anal. 149 (1997), 266-275.

[37] Thandapani E. and Ravi K., Bounded and monotone properties of solutions of second-order quasilinear forced difference equations. Comput. Math. Appl. 38 (1999), 113-121.

[38] Wong P. J. Y. and Agarwal R. P., Oscillation theorems for certain second order nonlinear difference equation. J. Math. Anal. Appl. 204 (1996), 813829.

[39] Xu Y. T. and Guo Z. M., Applications of a $Z_{p}$ index theory to periodic solutions for a class of functional differential equations. J. Math. Anal. Appl. 257 (2001), 189-205.

[40] Yu J. S. and Guo Z. M., On boundary value problems for a discrete generalized Emden-Fowler equation. J. Differential Equations 231 (2006), 18-31.

[41] Yu J. S., Long Y. H. and Guo Z. M., Subharmonic solutions with prescribed minimal period of a discrete forced pendulum equation. J. Dynam. Differential Equations 16 (2004), 575-586.

[42] Zhang F. Z., Matrix Theory:Basic Results and Techniques. Springer, New York (2011).

[43] Zhang R. Y., Wang Z. C. and Yu J.S., Necessary and sufficient conditions for the existence of positive solutions of nonlinear difference equations. Fields Inst. Commun. 42 (2004), 385-396.

[44] Zhou Z., Yu J. S. and Chen Y. M., Homoclinic solutions in periodic difference equations with saturable nonlinearity. Sci. China Math. 54 (2011), 83-93.

[45] Zhou Z., Yu J. S. and Guo Z. M., Periodic solutions of higher-dimensional discrete systems. Proc. Roy. Soc. Edinburgh (Section A) 134 (2004), 10131022.

[46] Zhou Z. and Zhang Q., Uniform stability of nonlinear difference systems. J. Math. Anal. Appl. 225 (1998), 486-500. 


\section{Haiping SHI}

Modern Business and Management Department

Guangdong Construction Vocational Technology Institute Guangzhou 510440, China

E-mail: shp7971@163.com

\section{Xia LiU}

Oriental Science and Technology College

Hunan Agricultural University

Changsha 410128, China

Science College

Hunan Agricultural University

Changsha 410128, China

E-mail: xia991002@163.com

Yuanbiao ZHANG

Packaging Engineering Institute

Jinan University

Zhuhai 519070, China

E-mail: abiaoa@163.com 